\title{
The adenovirus and the egg: A new approach to transgenesis
}

\author{
Alan R. Clarke
}

The past two decades have witnessed several revolutions in biological science, perhaps the most pervasive of which is the development of new approaches to access and genetically manipulate the germline of mice. These technologies now allow precise evaluation of the in vivo consequences of both gene overexpression and gene disruption. However, there are still numerous problems associated with transgenesis, particularly where the direct injection of DNA into oocytes is concerned. In this issue of Nature Biotechnology, Tsukui et al. ${ }^{\prime}$ describe a novel method for creating transgenic mice using adenovirus to transfer DNA sequences-in this case, the marker gene lac $Z$-directly into the genome of zona-free eggs.

Current approaches to generating transgenic animals fall into two broad categories: first, direct manipulation of the zygote or early embryo; and second, manipulation of pluripotent cells in vitro. Of the first category, the most widely used technique relies upon the direct physical injection of DNA into the pronucleus of mouse oocytes. Although relatively simple and efficient, this method does have two important caveats. These stem from the limited control over the process of DNA uptake, such that neither copy number nor site of integration can be controlled. This can have serious implications for the analysis of any transgenic strain, for example, leading to insertional inactivation at a random locus or leading to unpredicted patterns of transgene expression. The latter of these problems usually manifests itself in low levels of expression, which can in part be prevented by cointegration of transgenes ${ }^{2}$.

Several other methods have been used to introduce transgenes directly into the germline using zygotes or early embryos. Perhaps the most notorious of these was the report that sperm could act as a vehicle for

Alan $R$. Clarke is a royal society university research fellow at the department of pathology, University Medical School, Edinburgh, UK (aclarke@srv1.med.ed.ac.uk). transgenesis simply by mixing them with free $\mathrm{DNA}^{3}$. Difficulties with both unpredict-

\section{Current approaches to generating transgenic animals fall into two broad categories: first, direct manipulation of the zygote or early embryo; and second, manipulation of pluripotent cells in vitro.}

ed DNA rearrangements and low efficiency of DNA uptake have meant that this route has largely been ignored; however, this method has now been used to produce both transgenic cattle and swine 4 . A more widely

onic stem (ES) cells, in vitro. Although perhaps the most precise route to transgenesis, the ES cell system does suffer from several practical drawbacks in that it requires a substantial investment of time and effort, both in terms of bench work and also in the provision of appropriate culture facilities. It should also be stressed that the ES cell system is currently limited to manipulating the murine genome. To date, although considerable effort has been directed toward the generation of ES lines from other species, none has yet been used to successfully manipulate the germline of these species.

In vitro techniques are not limited to use of the ES cell system, as has been shown by the recent cloning of sheep by nuclear transfer from cell lines maintained in culture ${ }^{7}$. Given the current failure to produce ES cells from domestic species, applying the adenoviral approach described by Tsukui et al. to either oocytes or equivalent in vitro lineages may well represent a significant advance over current strategies.

The use of adenovirus as a vehicle for transgenesis has several clear benefits over current techniques. Perhaps the most important of these are its relative simplicity and the apparent ability to create single copy transgenics. However, a number of issues still need to be resolved before this technology can become universally applicable. For example, it remains to be proven that this technique will routinely deliver single-copy integration and efficient expression from a range of constructs and in a range of species. However, given the clear benefits of this technique in delivering a

adopted approach has been to use retroviral infection of early embryos, which has proved successful in a range of species ${ }^{5,6}$. Critically, this differs from the adenovirusmediated delivery described by Tsukui et al. in its ability to control both the extent of transgenesis within the embryo and also transgene copy number.

One approach to overcome some of these problems uses in vitro systems to characterize the nature of the introduced transgene before introduction to the germline. This is made possible by the ability to grow and manipulate pluripotent cells, such as embry- single transgene into the germline, adenovirus-mediated transgenesis should establish itself as an extremely valuable tool for the modern geneticist.

1. Tsukui, T., Kanegae, Y, Saito, I., and Toyoda, Y. 1996. Nature Biotechnology. 14:982-985.

2. Clark, A.J. et al. 1993. Phil. Trans. Roy. Soc. B. 339:225-232.

3. Lavitrano, M. et al. 1989. Cell 57:717-723.

4. Sperandio, S. et al. 1996. Anim. Biotechnol 7:59-77.

5. Rubenstein, J.L., Nicolas, J.F., and Jacob, F. 1986. Proc. Natt. Acad. Sci. USA 83:366-368.

6. Bowen, R.A., and Haskell. R.E. 1995. Mol. Reprod. Dev. 40:386-390.

7. Campbell, K.H.S., McWhir, J., Ritchie, W.A., and Wilmut, I. 1996. Nature 380:64-66. 